\title{
The Noncommutative Poisson Algebra of Classical and Quantum Mechanics
}

\author{
G. Morchio \\ Dipartimento di Fisica, Università di Pisa, \\ and INFN, Sezione di Pisa, Pisa, Italy \\ F. Strocchi \\ Scuola Normale Superiore, Pisa, Italy \\ and INFN, Sezione di Pisa
}

\begin{abstract}
The Lie-Rinehart (LR) algebra of a manifold $\mathcal{M}$, defined by the Lie structure of the vector fields, their action and their module structure on $C^{\infty}(\mathcal{M})$, is a common, diffeomorphism invariant, algebra for both classical and quantum mechanics. Its (noncommutative) Poisson universal enveloping algebra $\Lambda_{R}(\mathcal{M})$ contains a central variable $Z$ which relates the commutators to the Lie products; classical and quantum mechanics are its only factorial realizations, corresponding to $Z=i z, z=0$ and $z=\hbar$, respectively. In this form, canonical quantization appears therefore as a consequence of such a general geometrical structure. The regular factorial Hilbert space representations of $\Lambda_{R}(\mathcal{M})$ are, for $z \neq 0$, unitarily equivalent, apart from multiplicity, to one of the irreducible quantum representations, with $z=\hbar$, which are locally Schroedinger and in one to one correspondence with the unitary irreducible representations of the fundamental group of $\mathcal{M}$. For $z=0$, if $\operatorname{Diff}(\mathcal{M})$ is unitarily implemented, they are unitarily equivalent, up to multiplicity, to the representation defined by classical mechanics on $\mathcal{M}$.
\end{abstract}

Math. Sub. Class.: 81S10, 81Q70, 81R15, 53D17, 81R60, 81R10

Key words: Quantum mechanics on manifolds, Lie-Rinehart algebras, Noncommutative Poisson algebras, Canonical quantization, Classical mechanics 


\section{Introduction}

In a previous work 1] we discussed the formulation of Quantum Mechanics (QM) on a manifold $\mathcal{M}$ emphasizing the role of diffeomorphism invariance and of the associated Lie-Rinehart (LR) algebraic structure of the generators of $\operatorname{Diff}(\mathcal{M})$ as a module on $C^{\infty}(\mathcal{M})$. Such a construction relies on structures which are associated to the geometry of the (configuration) manifold $\mathcal{M}$ and are also at the basis of classical mechanics. It is therefore relevant to ask where and to which extent the classical and quantum approaches differ, starting from such common geometric structures.

In fact, we shall show that a (slight) generalization of the algebraic framework of Ref. [1] gives rise to an algebraic structure which is common to Classical $(\mathrm{CM})$ and quantum mechanics and actually has no other realizations. CM and QM will arise from the value of a central variable intrinsically provided by the common algebraic structure. It is worthwhile to stress that the symplectic geometry of the classical phase space is not an a priori common ingredient, but it rather characterizes one of the values of the central variable. Thus, the emerging structural relation between $\mathrm{CM}$ and QM, far from treating the second as a deformation of the classical symplectic structure, identifies both as realizations of the same algebra.

The analysis relies on the intrinsic LR algebraic structure associated to the configuration manifold $\mathcal{M}$ and to $\operatorname{Diff}(\mathcal{M})$ in terms of i) the algebra $C^{\infty}(\mathcal{M})$ generated by the $C^{\infty}$ real functions on $\mathcal{M}$ with compact support and by the constant functions, ii) the Lie algebra $\mathcal{L}(\mathcal{M})$ of $C^{\infty}$ vector fields $v$ of compact support, with Lie product $\{.,$.$\} , iii) the action of \mathcal{L}(\mathcal{M})$ on $C^{\infty}(\mathcal{M})$, denoted by $\{v, f\}$ and iv) the module structure of $\mathcal{L}(\mathcal{M})$ over $C^{\infty}(\mathcal{M})$, given by the LR product: $\forall f \in C^{\infty}(\mathcal{M}), \forall v \in \mathcal{L}(\mathcal{M}),(f, v) \rightarrow f \circ v \in \mathcal{L}(\mathcal{M})$, which is linear in both factors, associative in the first and satisfies $\{f \circ v, g\}=f\{v, g\}$.

The LR algebra $\mathcal{L}_{R}(\mathcal{M}) \equiv\left(C^{\infty}(\mathcal{M}), \mathcal{L}(\mathcal{M})\right)$ uniquely determines its non commutative Poisson universal enveloping algebra $\Lambda_{R}(\mathcal{M})$ (see Section 2). $\Lambda_{R}(\mathcal{M})$ has a Lie product $\{.,$.$\} , which extends that of \mathcal{L}_{R}(\mathcal{M})$ and an associative product, denoted by $\cdot$, whose symmetric (Jordan) part extends the partial product in $\mathcal{L}_{R}(\mathcal{M})$. The Lie product satisfies the Leibniz rule with respect to the associative product. No relation is assumed between the commutator $[A, B] \equiv A \cdot B-B \cdot A$ and the Lie product.

The noncommutative Poisson algebra $\Lambda_{R}(\mathcal{M})$ uniquely defines a notion of $(\operatorname{Diff}(\mathcal{M})$ invariant) noncommutative geometry of $(\mathcal{M}, \operatorname{Vect}(\mathcal{M}))$, only embodying the LR relations. Such a noncommutative geometry underlies both $\mathrm{CM}$ and $\mathrm{QM}$; in fact $\Lambda_{R}(\mathcal{M})$ is represented in both, with $[A, B]=0$ in the classical case, yielding the classical commutative Poisson algebra and the sym- 
plectic structure of the phase space; in the quantum case $[A, B]=i \hbar\{A, B\}$, yielding a realization of $\Lambda_{R}(\mathcal{M})$ through the Heisenberg algebra, with the LR product realized as the symmetric (Jordan) product, as recognized in Ref. [1] (see Section 4).

The main point of our analysis is that $\Lambda_{R}(\mathcal{M})$ contains a central variable $Z$ (a central sequence if the manifold is not compact), which relates the commutator to the Lie product:

$$
[A, B]=Z \cdot\{A, B\}, \quad[A, Z]=0=\{A, Z\}, \quad \forall A, B \in \Lambda_{R}(\mathcal{M}) .
$$

Under the standard reality assignment: $f^{*}=f, v^{*}=v, \forall f \in C^{\infty}(\mathcal{M})$, $v \in \mathcal{L}(\mathcal{M}), \Lambda_{R}(\mathcal{M})$ becomes a Poisson *-algebra and relations (1.1) imply that classical and quantum mechanics are its only "factorial realizations", i.e. such that the central variable $Z$ takes a definite value $\lambda \in \mathbf{C}$ (Sections 3,4).

This shows that the Dirac ansatz of canonical quantization, i.e. the proportionality of the commutators of positions and momenta to their classical Poisson brackets, has no alternative, in the sense that the only possibility for the commutator between variables in $\mathcal{L}_{R}(\mathcal{M})$ is given by their Lie product times a central variable. Hence, "quantization" is determined by the LR structure of $\mathcal{L}_{R}(\mathcal{M})$, the classical Poisson algebra and the Heisenberg algebra resulting as the unique alternatives.

Section 2 is devoted to the definition of the LR structure of $\mathcal{L}_{R}(\mathcal{M})$, to the analysis of its localization properties and to the construction of $\Lambda_{R}(\mathcal{M})$ as its unique non commutative Poisson universal enveloping algebra.

In Section 3, we exploit the localization properties of $\Lambda_{R}(\mathcal{M})$ induced by the LR local structure of $\mathcal{L}_{R}(\mathcal{M})$. This allows for the construction of variables which are locally central with respect to both the Lie and the commutator product. For a compact manifold they sum up to a (global) central variable $Z$, which relates the commutator to the Lie product, eq. (1.1). For non compact manifold, they give rise to a central sequence and therefore to an extension of $\Lambda_{R}(\mathcal{M})$ with a central variable $Z$ yielding eq. (1.1).

In Section 4, we consider the regular Hilbert space representations $\pi$ of the Poisson algebra $\Lambda_{R}(\mathcal{M})$, characterized by exponentiability of the representatives of the vector fields and of the central variable $Z$ to one parameter groups $U(\lambda v), U(\lambda Z)$, and by strong continuity of $\operatorname{Diff}(\mathcal{M})$ as automorphisms of the $C^{*}$-algebra generated by $\pi\left(C^{\infty}(\mathcal{M})\right)$ and by the $U(\lambda v), U(\lambda Z)$. Such representations are called factorial if the $\operatorname{Diff}(\mathcal{M})$ invariant elements of the center of the corresponding Von Neumann algebra are multiple of the identity. We show that i) for $z \neq 0$, the regular factorial Hilbert space representations of $\Lambda_{R}(\mathcal{M})$ are unitarily equivalent, apart from a multiplicity, to one of 
the irreducible quantum representations, with $z=\hbar$, classified in [1, which are locally Schroedinger and in one to one correspondence to the unitary irreducible representation of the fundamental group of $\mathcal{M}$; ii) for $z=0$, if $\operatorname{Diff}(\mathcal{M})$ is unitarily implemented, they are unitarily equivalent, up to a multiplicity, to the representation defined by classical mechanics on $\mathcal{M}$.

The above analysis shows the basic role of the LR geometry associated to the configuration manifold $\mathcal{M}$, which in the quantum case is somewhat hidden in the $C^{*}$-algebraic structure (since the Lie product is given by the commutator apart from the Planck constant), and in the classical case has nothing to do with the (abelian) algebraic structure. In fact, the LR geometry of $\operatorname{Diff}(\mathcal{M})$ provides a general notion of mechanical system, in terms of the Poisson universal enveloping algebra $\Lambda_{R}(\mathcal{M})$, classical and quantum mechanics being uniquely determined as its only factorial realizations. Such a strong implication is simply displayed in the case of $\mathbf{R}^{n}$, with the translation group playing the role of the diffeomorphisms.

This suggests a quite different approach to the relation between Classical and Quantum mechanics, with respect to phase space "quantization". The latter assumes as fundamental the classical canonical phase space with its symplectic diffeomorphism group, and associates $C^{*}$-algebras $\mathcal{A}_{\hbar}$ to it. Rather than trying to obtain Quantum mechanics from the symplectic structure of the classical phase space, our approach recognizes as fundamental the Lie-Rinehart noncommutative geometry of the configuration space $\mathcal{M}$ and of $V$ ect $(\mathcal{M})$, the classical symplectic space and the quantum mechanical state space resulting from the central classification of the same noncommutative Poisson algebra.

We shall adopt the following Notations:

$\mathcal{M}$ a connected $C^{\infty}$ manifold of dimension $d$, $\mathcal{O}$ any subset of $\mathcal{M}$ diffeomorphic to an open sphere,

$\operatorname{Diff}(\mathcal{M})$ the connected component of the identity of the group of diffeomorphisms of $\mathcal{M}$,

$\mathcal{L}(\mathcal{M}), \mathcal{L}(\mathcal{O})$ the Lie algebras of $C^{\infty}$ vector fields $v$ of compact support in $\mathcal{M}, \mathcal{O}$, with Lie product $\left\{v_{1}, v_{2}\right\}$

$g(\lambda v), \lambda \in \mathbf{R}, v \in \mathcal{L}(\mathcal{M})$, the associated one parameter groups, which exist by compactness of $\operatorname{supp} v$,

$\mathcal{G}(\mathcal{M})$ the subgroup of $\operatorname{Diff}(\mathcal{M})$ generated by the $g(\lambda v)$,

$\tilde{\mathcal{G}}(\mathcal{M})$ its universal covering group, which is uniquely associated to $\mathcal{L}(\mathcal{M})([2$, Theorem 8.1) and is generated by the elements of a neighborhood of the identity in $\mathcal{G}(\mathcal{M})$ and therefore by the one parameters groups $g(\lambda v)$,

$C_{0}^{\infty}(\mathcal{M}), C^{\infty}(\mathcal{B})$ the algebras generated by the $C^{\infty}$ real functions with compact support, respectively in $\mathcal{M}$ and in the open set $\mathcal{B}$,

$C^{\infty}(\mathcal{M})$ the algebra generated by $C_{0}^{\infty}(\mathcal{M})$ and by the constant functions. 


\section{The Lie-Rinehart noncommutative Poisson algebra of $\mathcal{M}$}

We recall the generalization of the notion of Poisson algebra to the noncommutative case [3] [4]:

Definition 2.1 A Poisson algebra $\Lambda$ is a real associative algebra, with product denoted by $A \cdot B$, and a (real) Lie algebra, with product denoted by $\{A, B\}$, satisfying the Leibniz rule

$$
\{A, B \cdot C\}=\{A, B\} \cdot C+B \cdot\{A, C\}
$$

Our analysis will be based on the following notion:

Definition 2.2 Given a (real) Lie algebra $\mathcal{L}$, with Lie product $\{.$, . $\}$, a Poisson enveloping algebra $\Lambda_{\mathcal{L}}$ of $\mathcal{L}$ is a Poisson algebra with a Lie algebra homomorphism $i: \mathcal{L} \rightarrow \Lambda_{\mathcal{L}}: \forall l_{1}, l_{2} \in \mathcal{L}$,

$$
i\left(\left\{l_{1}, l_{2}\right\}\right)=\left\{i\left(l_{1}\right), i\left(l_{2}\right)\right\} .
$$

A Poisson enveloping algebra $\Lambda_{\mathcal{L}}$ of a Lie algebra $\mathcal{L}$ will be called universal if, for any Poisson enveloping algebra $\Lambda_{\mathcal{L}}^{\prime}$, there is a unique homomorphism $\rho: \Lambda_{\mathcal{L}} \rightarrow \Lambda_{\mathcal{L}}^{\prime}$ of non commutative Poisson algebras, which satisfies $i_{\Lambda_{\mathcal{L}}^{\prime}}=\rho \circ i_{\Lambda_{\mathcal{L}}}$.

As in general for enveloping algebras, the uniqueness of the Poisson universal enveloping algebra (UEA) of a Lie algebra $\mathcal{L}$ follows immediately from the uniqueness of the homomorphism $\rho$. Its construction proceeds as in the Poincaré-Birkoff-Witt theorem by considering the tensor product polynomial algebra generated by $\mathcal{L}$ and by taking quotients with respect to the ideals generated by eqs. (2.1), (2.2). By construction $i$ is injective.

We recall the notion of Lie-Rinehart algebra [5]:

Definition 2.3 A real Lie-Rinehart (LR) algebra is a pair $\left(\mathcal{L}_{0}, \mathcal{L}\right)$, where $\mathcal{L}_{0}$ is a real commutative algebra, $\mathcal{L}$ is a (real) Lie algebra, with Lie product $\{.,$.$\} , which acts as derivations on \mathcal{L}_{0}, \forall f \in \mathcal{L}_{0}, v \in \mathcal{L}, f \rightarrow v(f)$ (hereafter $v(f)$ is denoted also by $\{v, f\}) ;\left(\mathcal{L}_{0}, \mathcal{L}\right)$ is equipped with a $\left(\right.$ LR $R$ product $\mathcal{L}_{0} \circ \mathcal{L} \subset$ $\mathcal{L}$, satisfying distributivity in both factors and, $\forall f, g \in \mathcal{L}_{0}, v, w \in \mathcal{L}$

$$
\begin{aligned}
& \{f \circ v, g\}=f\{v, g\}, \\
& f \circ(g \circ v)=(f g) \circ v,
\end{aligned}
$$




$$
\{v, f \circ w\}=\{v, f\} \circ w+f \circ\{v, w\} .
$$

A Lie-Rinehart algebra is said to have an identity, if the algebra $\mathcal{L}_{0}$ has an identity $\mathbf{1}$, which automatically satisfies $\{\mathbf{1}, v\}=0, \forall v \in \mathcal{L}$.

Clearly, $\mathcal{L}_{0}+\mathcal{L}$ is a Lie algebra, with Lie product $\{.,$.$\} and we define$

Definition 2.4 The Poisson universal enveloping algebra of a LR algebra $\left(\mathcal{L}_{0}, \mathcal{L}\right)$ with identity is the Poisson universal enveloping algebra of the Lie algebra $\mathcal{L}_{0}+\mathcal{L}$, with the additional relations $\forall f, g \in \mathcal{L}_{0}, \forall v \in \mathcal{L}$,

i) $i(\mathbf{1}) \cdot i(l)=i(l), \forall l \in\left(\mathcal{L}_{0}+\mathcal{L}\right)$

ii) $i(f g)=i(f) i(g)$,

iii) $i(f \circ v)=\frac{1}{2}(i(f) \cdot i(v)+i(v) \cdot i(f))$.

A Poisson UEA is not an enveloping algebra in the sense of envelops of Lie or of LR algebras [5], since no relation is assumed between the Lie product and the commutator. Obviously, the Poisson UEA of a LR algebra is isomorphic to the quotient of the Poisson UEA of $\mathcal{L}_{0}+\mathcal{L}$ with respect to the ideals defined by i)- iii). Existence and uniqueness of Poisson UEA follow as before.

$\mathcal{L}(\mathcal{M})$ acts as derivations on $C^{\infty}(\mathcal{M})$ and $\left(C^{\infty}(\mathcal{M}), \mathcal{L}(\mathcal{M})\right)$ is a LR algebra with LR product defined by eq. (2.3). The same holds for $\left(C_{0}^{\infty}(\mathcal{O}), \mathcal{L}(\mathcal{O})\right)$. $C^{\infty}(\mathcal{M})$ has an identity $1=1(x)$. Clearly, $\operatorname{Diff}(\mathcal{M})$ defines a group of automorphisms of $\left(C^{\infty}(\mathcal{M}), \mathcal{L}(\mathcal{M})\right)$ and of its Poisson UEA $\Lambda_{R}(\mathcal{M})$. The relation between the action of $\mathcal{G}(\mathcal{M})$ on $\Lambda_{R}(\mathcal{M})$ and the Lie product $\{.$, . $\}$ is

$$
(d / d \lambda) g_{\lambda v}(A)=\left\{v, g_{\lambda v}(A)\right\}, \quad \forall A \in \Lambda_{R}(\mathcal{M}),
$$

where $g_{\lambda v}$ denotes the one parameter subgroup of $\operatorname{Diff}(\mathcal{M})$ generated by $v$ and the derivative is taken in the $C^{\infty}$ topology.

Definition 2.5 The LR noncommutative Poisson algebra $\Lambda_{R}(\mathcal{M})$ of a manifold $\mathcal{M}$ is the Poisson universal enveloping algebra of the LR algebra $\left(C^{\infty}(\mathcal{M}), \mathcal{L}(\mathcal{M})\right)$.

We denote by $\Lambda(\mathcal{M})$ the Poisson UEA of $C^{\infty}(\mathcal{M})+\mathcal{L}(\mathcal{M})$ satisfying only condition i) of Definition 2.4.

With respect to $\Lambda_{R}(\mathcal{M})$, the Poisson UEA of $C^{\infty}(\mathcal{M})+\mathcal{L}(\mathcal{M})$ merely embodies the Lie relations between vector fields and functions on $\mathcal{M}$, so that it applies in general to the analysis of $\operatorname{Diff}(\mathcal{M})$. In particular, such a Lie algebra can be interpreted as the current algebra, which appears in all $N$-particle systems on $\mathcal{M}$ [6]. With respect to such a general structure, as discussed in Ref. [1], the 
mechanics of a particle on $\mathcal{M}$ requires the elimination of redundant degrees of freedom, which is provided by the LR relations between otherwise independent vector fields.

The algebra $\Lambda_{R}(\mathcal{M})$ can be interpreted as describing a particle on a manifold solely on the basis of general geometrical relations, which hold both in the quantum and in the classical case, since neither quantum commutation relations (as in Ref. 11), nor the vanishing of the commutator are assumed .

An important property of $\Lambda_{R}(\mathcal{M})$ is that there is a notion of localization in the following sense: an element $A \in \Lambda_{R}(\mathcal{M})$ has the compact set $K$ as a localization region, if it can be represented by a polynomial of elements of $\mathcal{L}_{0}(\mathcal{B})+\mathcal{L}(\mathcal{B})$, for some $\mathcal{B} \subset K$. In the case of non compact manifold $\mathcal{M}$, $\Lambda_{R}(\mathcal{M})$ is generated by the identity and by localized elements.

Proposition 2.6 If $A \in \Lambda_{R}(\mathcal{M})$ is localized in the compact set $K$ and $g \in$ $C^{\infty}(\mathcal{M})$ satisfies supp $g \cap K=\emptyset$, then

$$
i(g) \cdot A=A \cdot i(g)=0 .
$$

Therefore, by i), if $g(x)=1 \forall x \in K$, then

$$
i(g) \cdot A=A \cdot i(g)=A .
$$

Proof. If $A \in C^{\infty}(\mathcal{B}), \mathcal{B} \subset K$, the result follows from ii), trivially.

If $A=v \in \mathcal{L}(\mathcal{B})$, for $f \in C^{\infty}(\mathcal{B})$, with $f(x)=1, \forall x \in \operatorname{supp} v$, one has $f \circ v=v$ and therefore, as a consequence of ii), iii),

$$
i(g) \cdot i(v)=\frac{1}{2} i(g) \cdot\left[(i(f) \cdot i(v)+i(v) \cdot i(f)]=\frac{1}{2} i(g) \cdot i(v) \cdot i(f) .\right.
$$

By iii), and ii), $g \circ v=0$ implies that the r.h.s. is equal to $-\frac{1}{2} i(v) \cdot i(g f)=0$. By associativity of the product, eq. (2.7) extends to all $A \in \Lambda_{R}(\mathcal{M})$ localized in $K$.

\section{The relation between commutators and Lie products}

As remarked before, in a Poisson algebra there is no a priori relation between the commutator and the Lie product. As we shall prove in this section, for the LR Poisson algebra $\Lambda_{R}(\mathcal{M})$ the two products are not independent: for a compact manifold $\mathcal{M}$, there is a central variable which relates the commutator to the Lie product by eq. (1.1). In the non compact case, there are sequences 
which are central both in the Lie and in the commutator sense and give the same relations. The existence of central relations between commutators and Lie products has been recognized for strictly non commutative Poisson algebras which are prime, i.e. such that they do not have ideals which are divisors of zero [4]. This is not the case of $\Lambda_{R}(\mathcal{M})$, since in particular any pair of functions with disjoint supports generate (bilateral) ideals $I_{1}, I_{2}$ with $I_{1} \cdot I_{2}=0$ (as a consequence of Theorem 3.1 below). For brevity, in the following the injection $i$ will not be spelled out; $[A, B] \equiv A \cdot B-B \cdot A$ will denote the commutator.

Theorem 3.1 For a compact manifold $\mathcal{M}$, there exists a unique $Z \in \Lambda_{R}(\mathcal{M})$, such that, $\forall A, B \in \Lambda_{R}(\mathcal{M})$,

$$
[A, B]=Z \cdot\{A, B\}, \quad\{Z, A\}=0=[Z, A] .
$$

For a non compact manifold $\mathcal{M}$, there exists a sequence $Z_{n} \in \Lambda_{R}(\mathcal{M})$, such that $\forall A, B \in \Lambda_{R}(\mathcal{M})$,

$$
[A, B]=Z_{n} \cdot\{A, B\}, \quad\left\{Z_{n}, A\right\}=0=\left[Z_{n}, A\right], \forall n>\bar{n}(A, B) .
$$

Then, one can define an element $Z$, such that the Poisson algebra $\tilde{\Lambda}_{R}(\mathcal{M})$ generated by $\Lambda_{R}(\mathcal{M})$ and $Z$ satisfies eq. (3.1).

Proof. A crucial role is played by the property of $\operatorname{Diff}(\mathcal{M})$, by which the linear span of $\left\{C^{\infty}(\mathcal{M}), \mathcal{L}(\mathcal{M})\right\}$ contains $C^{\infty}(\mathcal{B}), \mathcal{B}$ any open set contained in a compact set $K$. In fact, for each open region $\mathcal{O}_{i}$, diffeomorphic to a sphere, by choosing a sphere $\mathcal{O}_{i}^{\prime}$ larger than $\mathcal{O}_{i}$, one can find $q_{i} \in C^{\infty}\left(\mathcal{O}_{i}^{\prime}\right), w_{i} \in \mathcal{L}\left(\mathcal{O}_{i}^{\prime}\right)$ such that $\left\{q_{i}, w_{i}\right\}(x)=1, \forall x \in O_{i}$. Then, for any $g_{i}$ with support contained in $\mathcal{O}_{i}$, in the LR algebra $\left(C^{\infty}(M), \mathcal{L}(\mathcal{M})\right)$ one has

$$
g_{i}=g_{i}\left\{q_{i}, w_{i}\right\}=\left\{q_{i}, g_{i} \circ w_{i}\right\},
$$

and, therefore, any $g \in C_{0}^{\infty}(\mathcal{M})$ has a decomposition

$$
g=\sum_{i} g_{i}=\sum_{i}\left\{q_{i}, g_{i} \circ w_{i}\right\}, \quad \operatorname{supp} g_{i} \subset \mathcal{O}_{i} .
$$

Then, by using the following identity, which holds in Poisson algebras, 4]

$$
[A, B] \cdot\{C, D\}=\{A, B\} \cdot[C, D],
$$

putting $Z_{g} \equiv \sum_{i}\left[q_{i}, g_{i} \circ w_{i}\right], \forall A, B \in \Lambda_{R}(\mathcal{M})$, one obtains

$$
[A, B] \cdot g=\sum_{i}[A, B] \cdot\left\{q_{i}, g_{i} \circ w_{i}\right\}=\{A, B\} \cdot Z_{g}
$$




$$
g \cdot[A, B]=Z_{g} \cdot\{A, B\} .
$$

The element $Z_{g}$ may depend on the choice of $g_{i}, q_{i}, w_{i}$ in the decomposition of $g$, eq. (3.4); however, by the same identity, for any $h$ of compact support one has

$$
Z_{g} \cdot h=\sum_{i}\left\{q_{i}, g_{i} \circ w_{i}\right\} \cdot Z_{h}=g \cdot Z_{h}=Z_{g}^{\prime} \cdot h,
$$

where, for given $g, Z_{g}^{\prime}$ corresponds to any other choice in the above construction.

Furthermore, for any $A$ such that $\{A, g\}=0$, in particular for all $A$ such that $g(x)=1, \forall x \in \operatorname{supp} A$, putting for brevity $p_{i} \equiv g_{i} \circ w_{i}$, using Leibniz rule and eq. (3.5), one has

$$
\begin{gathered}
\left\{\left[q_{i}, p_{i}\right], A\right\} \cdot g=\left(\left[\left\{q_{i}, A\right\}, p_{i}\right]+\left[q_{i},\left\{p_{i}, A\right\}\right]\right) \cdot g= \\
=\left(\left\{\left\{q_{i}, A\right\}, p_{i}\right\}+\left\{q_{i},\left\{p_{i}, A\right\}\right\}\right) \cdot Z_{g}=\left\{\left\{q_{i}, p_{i}\right\}, A\right\} \cdot Z_{g} .
\end{gathered}
$$

Hence,

$$
\left\{Z_{g} \cdot g, A\right\}=\sum_{i}\left\{\left[q_{i}, p_{i}\right], A\right\} \cdot g=\{g, A\} \cdot Z_{g}=0
$$

and

$$
\left[Z_{g} \cdot g, A\right]=\left[Z_{g}, A\right] \cdot g=\left\{Z_{g}, A\right\} \cdot Z_{g}=0 .
$$

In the case of a compact manifold, by taking $g=1$, condition i) implies eq. (3.1), with $Z \equiv Z_{1} ; Z_{1}$ is unique by eq. (3.7) and is a central variable by eqs. (3.8), (3.9).

For a non compact $\mathcal{M}$, by using Proposition 2.6, for any $g \in C^{\infty}(\mathcal{M}), Z_{g}$ is unique, by eq. (3.7) with $h=1$ in a region of localization of both $Z_{g}$ and $Z_{g}^{\prime}$. Moreover, for any increasing sequence of compact sets $K_{n}$ which cover $\mathcal{M}$, any sequence $g_{n} \in C^{\infty}(\mathcal{M})$ with compact support and $g_{n}(x)=1, \forall x \in K_{n}$, satisfies

$$
g_{n} \cdot A=A \cdot g_{n}=A, \quad n>\bar{n}(A),
$$

for all localized $A$, as a consequence of Proposition 2.6. Then, putting $Z_{n} \equiv$ $Z_{g_{n}}$, for $A$ localized in $K$ and $h$ such that $h \cdot A=A$, one has

$$
Z_{n} \cdot A=Z_{n} \cdot h \cdot A=Z_{n}^{\prime} \cdot h \cdot A=Z_{n}^{\prime} \cdot A,
$$

by eq. (3.7), i.e., for $n$ large enough, $Z_{n} \cdot A$ is independent of $n$ and of the construction of $Z_{n}$; again by eq. (3.7), $Z_{n} \cdot A$ is localized in $K$. Moreover, by eqs. (3.5), (3.8), (3.9), the sequences $Z_{n}$ satisfy eq. (3.2). 
Hence, one may consider the Poisson algebra $\tilde{\Lambda}_{R}(\mathcal{M})$ generated by $\Lambda_{R}(\mathcal{M})$ and by an element $Z$ defined by

$$
\begin{gathered}
Z \cdot A \equiv \lim _{n \rightarrow \infty} Z_{n} \cdot A=\lim _{n \rightarrow \infty} A \cdot Z_{n} \equiv A \cdot Z, \quad \text { for all localized } A, \\
Z \cdot \mathbf{1} \equiv Z \equiv \mathbf{1} \cdot Z, \quad\{Z, A\}=0, \quad \forall A \in \Lambda_{R}(\mathcal{M}) .
\end{gathered}
$$

Since, for localized $A, Z \cdot A$ is localized, $Z^{n} \cdot A \equiv Z^{n-1} \cdot(Z \cdot A)$ is recursively well defined; associativity and the Leibniz rule clearly hold and eq. (3.1) holds in $\tilde{\Lambda}_{R}(\mathcal{M})$.

In the above proof, conditions i)- iii) enter only after eq. (3.9), hence

Corollary 3.2 For the Poisson universal enveloping algebra $\Lambda_{L(\mathcal{M})}$ of the Lie algebra $L(\mathcal{M}) \equiv C^{\infty}(\mathcal{M})+\mathcal{L}(\mathcal{M})$ the commutators and the Lie products are related by

$$
\begin{gathered}
{[A, B] \cdot g=\{A, B\} \cdot Z_{g}, \quad A, B \in \Lambda_{L(\mathcal{M})}, g \in C^{\infty}(\mathcal{M})} \\
\left\{Z_{g} \cdot g, A\right\}=0=\left[Z_{g} \cdot g, A\right], \quad \text { if }\{A, g\}=0 .
\end{gathered}
$$

Furthermore, since only i) has been used in the case of compact $\mathcal{M}$, one has

Corollary 3.3 For a compact $\mathcal{M}$, Theorem 3.1 holds for $\Lambda(\mathcal{M})=$ the Poisson UEA of the Lie algebra $L(\mathcal{M})$, satisfying condition i) of Definition 2.4.

For the implications of the above results on Classical and Quantum Mechanics, we note that a notion of reality in $\Lambda_{R}(\mathcal{M})$ is automatically given if the elements of $C^{\infty}(\mathcal{M})+\mathcal{L}(\mathcal{M})$ are taken as real:

$$
f^{*}=f, \quad v^{*}=v .
$$

The $^{*}$ operation obviously extends to a (real linear) involution on $\Lambda_{R}(\mathcal{M})$, with $\{A, B\}^{*}=\left\{A^{*}, B^{*}\right\},(A B)^{*}=B^{*} A^{*}$ and therefore $Z^{*}=-Z$. Then, $\Lambda_{R}(\mathcal{M})$ becomes a Poisson ${ }^{*}$-algebra (Definition 4.1 below) and as such will be understood in the following.

"Factorial realizations" of $\Lambda_{R}(\mathcal{M})$ are defined by homomorphisms $\pi_{z}$ obtained by taking the quotient of $\Lambda_{R}(\mathcal{M})$ with respect to the ideal generated by $Z^{*} Z-z^{2}, z \geq 0$. (For Hilbert space representations see Section 4).

For $z=0$, one obtains the commutative Poisson algebra generated by $C^{\infty}(\mathcal{M})$ and by the polynomials of the $C^{\infty}$ vector fields, with the natural module structure of $\mathcal{L}(\mathcal{M})$ on $C^{\infty}(\mathcal{M})$, i.e., substantially, the symplectic structure of 
classical mechanics (see Section 4). Such an algebra is also very close to the Lie algebroid advocated as the algebraic structure of classical mechanics 7 . For $z^{2} \neq 0, \pi_{z}(Z)=\iota z, \iota^{2}=-1$ and there is an isomorphism $\varphi$, mapping the real Poisson involutive algebra into the complex algebra generated by $C^{\infty}(\mathcal{M})$ and by the generalized momenta $T_{v}$ associated to the vector fields of $\mathcal{L}(\mathcal{M})$, satisfying

$$
\left[T_{v}, T_{w}\right]=i z T_{\{v, w\}}, \quad\left[T_{v}, f\right]=i z\{v, f\}, \quad z \geq 0
$$

with $\varphi(f)=f, \varphi(v)=T_{v}, \varphi(\iota)=i$. This is the (unbounded) LR quantum algebra introduced in Ref.[1]. Such an isomorphism between $\pi_{z}\left(\Lambda_{R}(\mathcal{M})\right)$ and the above complex algebra offers an explanation of the occurrence of a complex structure in the standard formulation of quantum mechanics, whereas no complex structure is needed for the formulation of classical mechanics.

In conclusion, the Poisson UEA $\Lambda_{R}(\mathcal{M})$, with the reality notion (3.13), is common to classical and quantum mechanics, which can be characterized as its only factorial realizations, in the sense defined above.

The central relation between commutators and Lie products, given by Corollaries 3.2, 3.3, are independent of the LR relations and therefore apply to other geometric structures which can be associated to $\operatorname{Diff}(\mathcal{M})$, without including the LR relations. In fact, the Lie algebra $L(\mathcal{M})=C^{\infty}(M)+\mathcal{L}(M)$ can be interpreted as the current Lie algebra on $\mathcal{M}$ generated by the charge densities $\rho(f), f \in C^{\infty}(\mathcal{M})$ and the currents $j(v), v \in \mathcal{L}(M)$, with

$$
\{\rho(f), \rho(g)\}=0, \quad\{j(v), \rho(f)\}=\rho(\{v, f\}), \quad\{j(v), j(w)\}=j(\{v, w\}),
$$

for all $f, g \in C^{\infty}(\mathcal{M}), v, w \in \mathcal{L}(\mathcal{M})$. Hence, $\Lambda(\mathcal{M})$ is identified with the Poisson UEA of the current Lie algebra with the additional relation i) of Definition 2.4, $i(\rho(1)) \cdot A=A, \forall A \in \Lambda_{L(\mathcal{M})}$. Such a relation holds for the $N$-particle representations of the current Lie algebra introduced by Goldin [6], provided $\rho$ is identified with the Goldin charge density divided by $N$.

With the above identifications, the Poisson algebra $\Lambda(\mathcal{M})$ describes both the classical and the quantum current algebra and for compact $\mathcal{M}$, by Corollary 3.3, these are the only factorial realizations, in the above sense. The LR relations are not satisfied in the current algebra, where in particular the algebra generated by the $\rho(f)$ is a free abelian algebra.

As it is clear from the proof of Theorem 3.1, the central variable relation between commutators and Lie products follows in general from the existence of an identity which can be written as a sum of Lie products. As a physically relevant example, we consider the Lie algebra of the canonical variables 
in $\mathbf{R}^{n}$, defined by the following Lie products

$$
\left\{q_{i}, q_{j}\right\}=0=\left\{p_{i}, p_{j}\right\}, \quad\left\{q_{i}, p_{j}\right\}=\delta_{i j} \mathbf{1}, \quad\left\{q_{i}, \mathbf{1}\right\}=0=\left\{p_{i}, \mathbf{1}\right\},
$$

henceforth denoted by $\mathcal{L}_{c}$. The construction of Theorem 3.1 simplifies and the corresponding Poisson UEA $\Lambda_{c}$, defined with the condition i) of Definition 2.4, $i(\mathbf{1}) \cdot A=A$, contains a central variable $Z$, such that

$$
\begin{gathered}
{\left[q_{i}, q_{j}\right]=Z\left\{q_{i}, q_{j}\right\}=0, \quad\left[p_{i}, p_{j}\right]=Z\left\{p_{i}, p_{j}\right\}=0,} \\
{\left[q_{i}, p_{j}\right]=Z\left\{q_{i}, p_{j}\right\}=Z \delta_{i j} .}
\end{gathered}
$$

Thus, the commutator between any pair of canonically conjugated variables is a central variable in $\Lambda_{c}$ and coincides with $Z$.

The Poisson algebra $\Lambda_{c}$ describes both classical and quantum mechanics, the two realizations corresponding, as above, to the quotients of $\Lambda_{c}$ with respect to the ideals generated by $Z^{*} Z-z^{2}, z \geq 0$, with, respectively, $z=0$ and $z=\hbar$.

\section{Representations of $\Lambda_{R}(\mathcal{M})$. Classical and Quan- tum Mechanics}

The Poisson algebra $\Lambda_{R}(\mathcal{M})$ involves unbounded variables and the analysis of its representations is conveniently done in terms of associated normed * algebras and $C^{*}$-algebras. For this purpose, we introduce the following notions.

Definition 4.1 A Poisson *algebra $\Lambda$ is a (noncommutative) Poisson algebra, with a (real linear) involution *, satisfying $\{A, B\}^{*}=\left\{A^{*}, B^{*}\right\}$, $(A \cdot B)^{*}=B^{*} \cdot A^{*}$.

$A$ representation $\pi$ of a Poisson *algebra $\Lambda$ in a Hilbert space $\mathcal{H}$ is a homomorphism of $\Lambda$ into a Poisson *-algebra of operators in $\mathcal{H}$ (with both the operator product and a Lie product $\{.,$.$\} satisfying the Leibniz rule), having$ a common invariant dense domain $D$ on which

$$
\pi(A \cdot B)=\pi(A) \pi(B), \quad \pi(\{A, B\})=\{\pi(A), \pi(B)\}, \quad \pi\left(A^{*}\right)=\pi(A)^{*} .
$$

Proposition 4.2 In a representation $\pi$ of (the Poisson *algebra) $\Lambda_{R}(\mathcal{M})$ in a Hilbert space $\mathcal{H}, \pi(f)$ and $\pi(v), f \in C^{\infty}(\mathcal{M}), v \in \mathcal{L}(\mathcal{M})$ are strongly continuous on $D$ in the $C^{\infty}$ topology of $C^{\infty}(\mathcal{M})$ and $\mathcal{L}(\mathcal{M})$.

Furthermore, eq. (2.6) holds for $\pi\left(g_{\lambda v}(A)\right), A=f, v$, with the derivative taken in the strong topology. 
Proof. Since for $|\lambda|>\|f\|_{\infty}, f-\lambda$ is invertible in $C^{\infty}(\mathcal{M}),\|\pi(f)\| \leq\|f\|_{\infty}$. Moreover, any compact set $K \subset \mathcal{M}$ can be covered by a finite number of open sets $\mathcal{O}$ homeomorphic to spheres, so that, by a corresponding decomposition of unity, any vector field $v$ can be written as a linear combination $v=\sum_{i}^{d} f_{i} w_{i}, f_{i} \in C^{\infty}(\mathcal{M})$. Then, $v_{n} \rightarrow v$ in the $C^{\infty}$ topology implies that the corresponding $f_{i}^{(n)}$ converge $f_{i}$ in $C^{\infty}(\mathcal{O})$ and, by the LR relation $\pi\left(f_{i} w_{i}\right)=\pi\left(f_{i}\right) \pi\left(w_{i}\right)+\pi\left(w_{i}\left(f_{i}\right)\right) Z, \pi\left(v_{n}\right)$ converge strongly to $\pi(v)$ on $D$. This also implies that $\pi\left(g_{\lambda v}(A)\right)$ is strongly differentiable in $\lambda$ and eq. (2.6) holds for $\pi\left(g_{\lambda v}(A)\right)$.

Definition 4.3 A representation $\pi$ of $\Lambda_{R}(\mathcal{M})$ in a complex Hilbert space $\mathcal{H}$ is called regular if $\pi\left(C_{0}^{\infty}(\mathcal{M})\right) \neq 0$ and

i) (exponentiability) $D$ is invariant under the $C^{*}$-algebra $\mathcal{A}(\mathcal{M})_{\pi}$ generated by $\pi\left(C^{\infty}(\mathcal{M})\right.$ ) and by one parameter unitary groups $U(\lambda v), U(\lambda Z), \lambda \in \mathbf{R}$, generated by $T_{v} \equiv \pi(v)$ and $-i \pi(Z)$, respectively,

ii) (diffeomorphism invariance) the elements $g_{\lambda v} \in \mathcal{G}(\mathcal{M})$ define strongly continuous automorphisms of $\mathcal{A}(\mathcal{M})_{\pi}$ by

$$
g_{\mu w}: \pi(f) \rightarrow \pi\left(g_{\mu w} f\right), \quad U(\lambda v) \rightarrow U\left(\lambda g_{\mu w}(v)\right), \quad U(\lambda Z) \rightarrow U(\lambda Z) .
$$

Property ii) states the exponentiability of the derivation (2.6) in the representation $\pi$; it is implied by i) if $z \neq 0$ (see below).

Proposition 4.4 In a regular representation $\pi$ of $\Lambda_{R}(\mathcal{M})$ ), the one-parameter unitary groups $U(\lambda v), U(\lambda Z)$ satisfy

$$
[U(\lambda v), U(\lambda Z)]=0, \quad[\pi(f), U(\lambda Z)]=0 .
$$

Proof. In fact, one has on $D$

$$
i(d / d \lambda)[U(\lambda v) \pi(Z) U(-\lambda v)]=U(\lambda v)\left[T_{v}, \pi(Z)\right] U(-\lambda v)=0,
$$

i.e. $[U(\lambda v), \pi(Z)]=0$; therefore $(d / d \lambda) U(\lambda Z) U(\mu v) U(\lambda Z)^{-1}=0$. Similarly for $\pi(f)$ and eqs. (4.2) follow.

Since $\Lambda_{R}(\mathcal{M})$ has both an associative product and a Lie product related to $\operatorname{Diff}(\mathcal{M})$ by eq. $(2.6)$, a natural role is played by elements which are central with respect to both.

Definition 4.5 A regular representation $\pi$ of $\Lambda_{R}(\mathcal{M})$ is called factorial if the elements of the center $\mathcal{Z}_{\pi}$ of the Von Neumann algebra $\mathcal{A}(\mathcal{M})_{\pi}^{\prime \prime}$ which are invariant under Diff $(\mathcal{M})$ are multiples of the identity. 
Classical and Quantum Mechanics are examples of regular (factorial) representations of $\Lambda_{R}(\mathcal{M})$.

A) Quantum Mechanics. As discussed in Ref. [1], QM on a manifold $\mathcal{M}$, with $\hbar=1$, is described by the $L R$ regular representations $\pi$ of the crossed product $\Pi(\mathcal{M}) \equiv C^{\infty}(\mathcal{M}) \times \tilde{\mathcal{G}}(\mathcal{M})$, i.e. such that $\pi\left(C_{0}^{\infty}(\mathcal{M})\right) \neq 0$ and i) $\pi(U(\lambda v))$ are strongly continuous in $\lambda$, ii) their generators $T_{v}$ have a common dense domain $D$ invariant under $\Pi(\mathcal{M})$, satisfy the Lie algebra relations and the LR relations, $\forall f \in C^{\infty}(\mathcal{M}), v, w \in$ $\mathcal{L}(\mathcal{M})$

$$
\left[T_{v}, T_{w}\right]=i T_{\{v, w\}}, \quad T_{f \circ v}=\frac{1}{2}\left(f T_{v}+T_{v} f\right) .
$$

By Propositions 4.2, 4.6, $\pi(U(\lambda v))$ are also strongly continuous in the $C^{\infty}$ topology of the vector fields. The classification of irreducible such representations [1, implies, by their locally Schroedinger property, that $D$ can be chosen invariant under the generators $T_{v}$ and therefore one actually gets a regular representation of $\Lambda_{R}(\mathcal{M})$ with $Z=i$. The automorphisms $g_{\lambda v}$ are implemented by $U(\lambda v)$ and property ii) of Definition 4.3 follows; the representation of $\Lambda_{R}(\mathcal{M})$ is factorial if $\pi$ is irreducible.

More generally, the above operators $f$ and $T_{\hbar v}$ define regular representations of $\Lambda_{R}(\mathcal{M})$ with $Z=i \hbar$. Such representations are therefore classified by $\hbar$ and the unitary representations of the fundamental group $\pi_{1}(\mathcal{M})$ [1]; they shall be called the quantum representations of $\Lambda_{R}(\mathcal{M})$.

B) Classical Mechanics. Given the cotangent bundle $T^{*} \mathcal{M}$, described by local coordinates $(x, p)$, with $x \in \mathcal{M}$ and $p_{i}$ the coordinates in the basis dual to $\partial / \partial x_{i}$, we consider the Hilbert space $L^{2}\left(T^{*} \mathcal{M}, d x d p\right)$ and the representation $\pi_{C}$ of $\Lambda_{R}(\mathcal{M})$, with $Z=0$, by multiplication operators on $D=C_{0}^{\infty}\left(T^{*} \mathcal{M}\right)$, $\forall f \in C^{\infty}(\mathcal{M}), \forall v=\sum_{i} g_{i}(x) \partial / \partial x_{i}, \operatorname{supp} v \subseteq \mathcal{O},(\forall \mathcal{O}$ homeomorphic to an open sphere),

$$
\pi_{C}(f)=f(x), \quad \pi_{C}(v)=\sum_{i} g_{i}(x) p^{i} \equiv T_{v}, \quad U(\lambda v)=e^{-i \lambda \sum_{i} g_{i}(x) p^{i}} .
$$

The Lie product on $\Lambda_{R}(\mathcal{M})$ is given by the standard Poisson brackets $\{., .\}_{C}$ on $T^{*} \mathcal{M}$. The automorphisms $g_{\lambda v}, g_{\lambda v}(f)(x)=f\left(g_{\lambda v}^{-1}(x)\right), g_{\lambda w}\left(T_{v}\right)=T_{g_{\lambda w}(v)}$, satisfy eq. (2.6), extend to the $U(\lambda v)$ and are unitarily represented. Hence, $\pi_{C}$ is a regular representation of $\Lambda_{R}(\mathcal{M})$, (actually it is factorial), with $Z=0$. It shall be referred to as the classical canonical representation of $\Lambda_{R}(\mathcal{M})$.

In the following, we show that the regular factorial representations of $\Lambda_{R}(\mathcal{M})$ are quasi equivalent to either one of the quantum irreducible representations or (in separable Hilbert spaces) to the classical canonical representation. 
Proposition 4.6 In a regular factorial representation $\pi$ of $\Lambda_{R}(\mathcal{M})$ one has i) $U(\lambda Z)=e^{-i \lambda z} \mathbf{1}, z \in \mathbf{R}$; apart from an antiunitary transformation leaving $\Lambda_{R}(\mathcal{M})$ pointwise invariant, one can take $z \geq 0$,

ii) the one parameter groups $U(\lambda v)$ are strongly continuous in $v$ in the $C^{\infty}$ topology of the vector fields and

$$
U(\lambda v) U(\mu w)=U\left(\mu g_{\lambda z v}(w)\right) U(\lambda v), \quad U(\lambda v) f=g_{\lambda z v}(f) U(\lambda v),
$$

$g_{\lambda z v}(\cdot)$ denoting the action of the one parameter group of $\mathcal{G}(\mathcal{M})$ generated by $\lambda z v$.

Proof. $\quad$ By eq. (4.2), $U(\lambda Z)$ belongs to $\mathcal{Z}_{\pi}$ and, by eq. (4.1), is invariant under $\operatorname{Diff}(\mathcal{M})$; then, $U(\lambda Z)=e^{-i \lambda z} \mathbf{1}$, by strong continuity in $\lambda$. For the antiunitary transformation see Section 3.

Moreover, by using Proposition 4.2, $\forall A \in C^{\infty}(\mathcal{M})+\mathcal{L}(\mathcal{M})$ one has

$$
\begin{gathered}
(d / d \lambda)\left[U(-\lambda v) \pi\left(g_{\lambda z v}(A)\right) U(\lambda v)\right]= \\
=U(-\lambda v)\left(i\left[T_{v}, \pi\left(g_{\lambda z v}(A)\right)\right]+\left\{z T_{v}, \pi\left(g_{\lambda z v}(A)\right)\right\}\right) U(\lambda v)=0 .
\end{gathered}
$$

Then,

$$
U(-\lambda v) \pi\left(g_{\lambda z v}(A)\right) U(\lambda v)=A
$$

and eqs. (4.3) follow, by exponentiability. Finally, $\forall \Psi \in D$, eq. (4.3) implies $(d / d \lambda) \Psi_{n}(\lambda) \equiv(d / d \lambda)\left[U\left(\lambda v_{n}\right) U(-\lambda v)\right] \Psi=U\left(\lambda v_{n}\right) U(-\lambda v)\left(T_{g_{-\lambda z v}\left(v_{n}\right)}-T_{v}\right) \Psi$ and $T_{g_{-\lambda z v}\left(v_{n}\right)} \Psi=T_{g_{-\lambda z v} g_{\lambda z v_{n}}\left(v_{n}\right)} \Psi$ converges strongly to $T_{v} \Psi$, uniformly in $\lambda$ (bounded), by Proposition 4.2; hence, $\Psi_{n}(\lambda)=\Psi+\int_{0}^{\lambda} d \lambda^{\prime}\left(d / d \lambda^{\prime}\right) \Psi_{n}\left(\lambda^{\prime}\right) \rightarrow \Psi$.

Theorem 4.7 The regular factorial representations $\pi$ of $\Lambda_{R}(\mathcal{M})$ are classified by the value $i z$ of the central variable $Z$ and

1) for $z \neq 0$, each of them is unitarily equivalent, apart from a multiplicity, to one of the irreducible quantum representations defined above by a LR regular irreducible representation of the crossed product $C^{\infty}(\mathcal{M}) \times \tilde{\mathcal{G}}(\mathcal{M})$, with $z=\hbar$, and therefore in one to one correspondence with the unitary representations of $\pi_{1}(\mathcal{M})$

2) for $z=0$, for separable representation space $\mathcal{H}$, they are quasi equivalent to the classical canonical representation, actually, unitarily equivalent, up to a multiplicity, if Diff $(\mathcal{M})$ is unitarily implemented. 
Proof. For $z \neq 0$, by eqs. (4.3), $\pi(f), f \in C^{\infty}(\mathcal{M})$ and the $U\left(\lambda z^{-1} v\right), v \in$ $\mathcal{L}(\mathcal{M})$, provide a representation of the crossed product $\Pi(\mathcal{M})$, which is LR regular by Definition 4.3, i).

Since the automorphisms $g_{\lambda v}$ are unitarily implemented by the $U\left(\lambda z^{-1} v\right)$, the center of $\Pi(\mathcal{M})^{\prime \prime}$ is automatically pointwise invariant under $\mathcal{G}(\mathcal{M})$ and, therefore, factoriality in the sense of Definition 4.5 implies factoriality of the representation of the crossed product. Hence, the representation is a sum of copies of one irreducible representation of $\Pi(\mathcal{M})$; the latter are classified by the unitary irreducible representation of $\pi_{1}(\mathcal{M})$, Ref. [1] . As remarked before, each LR regular irreducible representation of $\Pi(\mathcal{M})$ defines a regular factorial representation of $\Lambda_{R}(\mathcal{M})$.

For $z=0$, by eqs. (4.3), the $C^{*}$-algebra $\mathcal{A}(\mathcal{M})_{\pi}$ generated by $\pi\left(C^{\infty}(\mathcal{M})\right)$ and the $U(\lambda v)$ is abelian; by separability, the representation space can therefore be identified with a sum of $L^{2}$ spaces with finite measures $\sigma_{n}$ over the spectrum $\Sigma$ of $\mathcal{A}(\mathcal{M})_{\pi}$, whose points $\xi$, apart from sets of zero measure for all $\sigma_{n}$, are identified as multiplicative linear functionals which by regularity can be characterized as follows: $\forall f \in C^{\infty}(\mathcal{M}), \xi(f)=f\left(x_{\xi}\right)$, and $\forall v=\sum_{i} g_{i}(x) \partial / \partial x_{i}$, $\operatorname{supp} v \subseteq \mathcal{O},(\forall \mathcal{O}$ homeomorphic to an open sphere)

$$
\xi(v)=\sum_{i} g_{i}\left(x_{\xi}\right) p_{\xi}^{i}, \quad p_{\xi}^{i} \equiv \xi\left(\partial / \partial x_{i}\right), \quad \xi(U(\lambda v))=\exp \left(i \sum_{i} g_{i}\left(x_{\xi}\right) p_{\xi}^{i}\right),
$$

i.e. the point $\xi$ is identified by local coordinates $(x, p)$ in $T^{*} \mathcal{M}$. Then, apart from a set of zero $\sigma_{n}$ measure, $\Sigma$ can be identified with a subset of $T^{*} \mathcal{M}$, by a continuous map, so that $\sigma_{n}$ defines a finite Borel measure $\mu_{n}$ on $T^{*} \mathcal{M}$ and the representation space $\mathcal{H}$ may be taken as $\sum_{n} L^{2}\left(T^{*} \mathcal{M}, d \mu_{n}\right)$.

The set of points $I_{0} \equiv\{(x, 0), x \in \mathcal{M}\}$ is invariant under $\operatorname{Diff}(\mathcal{M})$ and it has zero $\mu_{n}$-measure, since, otherwise, it would define a subrepresentation of $\Lambda_{R}(\mathcal{M})$, which violates the validity of eq. (2.6), as stated by Proposition 4.2. The action of $\operatorname{Diff}(\mathcal{M})$ on $T^{*} \mathcal{M}$ is transitive on the points $(x, p), p \neq 0$; moreover, for each point $(x, p), p \neq 0$, there are $2 d$ vector fields $v_{i}$, such that $\lambda_{i}, i=1, \ldots 2 d,\left|\lambda_{i}\right|<2 \varepsilon$, define local coordinates for $g_{\lambda_{i} v_{i}}(x, p)$. If $I \subseteq T^{*} \mathcal{M}$ is a Borel set of zero Lebesgue measure, contained in a neighborhood of $(\bar{x}, \bar{p})$, one has (by Fubini theorem)

$$
\int_{\left|\lambda_{i}\right|<\varepsilon} \Pi d \lambda_{i} \int d \mu \chi_{I, g_{\lambda_{i} v_{i}}}=\int d \mu \int_{\left|\lambda_{i}\right|<\varepsilon} \Pi d \lambda_{i} \chi_{I, g_{\lambda_{i} v_{i}}}=0, \quad d \mu \equiv \sum_{n} 2^{-n} d \mu_{n},
$$

where $\chi_{I}$ is the characteristic function of $I$ and $\chi_{I, g_{\lambda_{i} v_{i}}}(x, p) \equiv \chi_{I}\left(g_{\lambda_{i} v_{i}}^{-1}(x, p)\right)$. Then, $\int d \mu \chi_{I}\left(g_{\lambda_{i} v_{i}}^{-1}(x, p)\right)=0$, a.e. in $\lambda_{i}$ and, since the projection $E_{I}$ and 
$g_{\lambda v}\left(E_{I}\right)=E_{g_{\lambda v}^{-1}(I)}$ are isomorphic, $\int d \mu \chi_{I}=0$. Therefore, one has a representation of $\Lambda_{R}(\mathcal{M})$, as an abelian algebra, quasi equivalent to the classical canonical representation. Conversely, if $\mu(I)=0$ and $I$ has non zero Lebesgue measure, $\int_{\left|\lambda_{i}\right|<\varepsilon} \Pi d \lambda_{i} \chi_{I, g_{\lambda_{i} v_{i}}}(x, p)>\delta>0$, for $(x, p)$ in an open set $\mathcal{O}_{I}$ and therefore the above equation implies $\mu\left(\mathcal{O}_{I}\right)=0$; by the transitivity of $\operatorname{Diff}(\mathcal{M})$, $d \mu=0$. Therefore, $d \mu$ is equivalent to the Lebesgue measure.

The Lie product on $\pi\left(\Lambda_{R}(\mathcal{M})\right)$ is completely determined by its restriction to $C^{\infty}(\mathcal{M})+\mathcal{L}(\mathcal{M})$, which, by Proposition 4.2 satisfies eq. (2.6) and locally one has, $\forall A(x, p) \in C^{\infty}(\mathcal{M})+\mathcal{L}(\mathcal{M})$,

$$
\begin{gathered}
\left\{\sum_{i} g_{i}(x) p^{i}, A(x, p)\right\}=\left.(d / d \lambda) A\left(g_{\lambda v}^{-1}(x, p)\right)\right|_{\lambda=0}= \\
=\sum_{i}\left(-\frac{\partial A(x, p)}{\partial x_{i}} g_{i}(x)+\frac{\partial A(x, p)}{\partial p^{i}} \frac{\partial g_{j}(x)}{\partial x_{i}} p^{j}\right)=\left\{\sum_{i} g_{i}(x) p^{i}, A(x, p)\right\}_{C} .
\end{gathered}
$$

Thus, the representation of $\Lambda_{R}(\mathcal{M})$, as Poisson algebra, is given by the classical canonical representation, up to multiplicities. If $\operatorname{Diff}(\mathcal{M})$ is unitarily implemented, the multiplicity of the representation is $\mu$ a.e. constant and therefore it is a multiple of the classical canonical representation.

\section{References}

[1] Morchio, G., Strocchi, F.: Quantum mechanics on manifolds and topological effects. Lett. Math. Phys. 82, 219-236 (2007)

[2] Milnor, J.: Remarks on infinite-dimensional Lie groups. In: DeWitt, B.S., Stora, R. (eds.) Relativity, groups and Topology II., Elsevier (1984)

[3] Flato M., Gerstenhaber, M., Voronov, A.A.: Cohomology and Deformation of Leibniz Pairs. Lett. Math. Phys. 34, 77-90 (1995)

[4] Farkas, D.R., Letzer, G.: Ring theory from symplectic geometry. J. Pure Appl. Algebra 125, 155-190 (1998)

[5] Rinehart, G.: Differential forms for general commutative algebras. Trans. Amer. Math. Soc. 108, 195-222 (1963)

[6] Goldin, G.A.: Lectures on diffeomorphism groups in quantum physics, in Contemporary Problems in Mathematical Physics, Govaerts, J., Hounkonnou, N., Msezane, A.Z., eds., World Scientific 2006, p.1-93 
[7] Landsman, N.P.: Lie Groupoids and Lie algebroids in Physics and noncommutative geometry. J. Geom. Phys. 56, 24-54 (2006) 